\title{
Study on the Protection and Inheritance of Grand Song of Dong Minority
}

\author{
Guangtan Qi \\ Baicheng Normal University \\ Baicheng, China
}

\begin{abstract}
Since the economic development of China, the construction of spiritual civilization has been risen to higher level. The modern civilization construction progress is mainly based on the national traditional culture to improve the national cultural connotation on this basis. Thereinto, with the continuous development of national social environment, the aesthetic idea of people has been greatly improved which causes significant changes of some folk-customs. The grand song of Dong Minority is closely bonded together with the society during development and its main purposes are the protection and inheritance of grand song of Dong Minority. However, the grand song of Dong minority cannot better adapt to the social development which requires taking necessary methods to conduct effective protection so as to achieve the purpose of inheritance on this basis and lay a good foundation for the inheritance of traditional culture of China.
\end{abstract}

Keywords-grand song of Dong minority; protection; inheritance

\section{INTRODUCTION}

As the national traditional culture, the grand song of Dong minority has years of history and this traditional national art possesses relatively high aesthetic value and social value no matter on the aspect of form or content. Therefore, it was listed as tangible cultural heritage of China. In addition, there have been significant changes in the grand song of Dong minority of China during the development process which is mainly related to the prevailing circumstances. The natural environment, aesthetic consciousness and social customs, etc of Dong minority determine the form and content of grand song of Dong minority to a large extent and carry the development and inheritance of Dong minority culture which possess relatively high realistic functions to a large extent. Such aspects not only can play key functions to the protection and inheritance of grand song of Dong minority of China but also can lay the good foundation for the future social spiritual civilization construction of China.

\section{INTRODUCTION TO GRAND SONG OF DONG MINORITY}

Grand song of Dong minority is mainly originated from the Spring and Autumn Period and the Warring States Period and has thousands of years of development history. It is mainly in the form of folk chorus with relatively complex structure and it possesses special singing mode and requires singing of multiple people. The singers shall be strictly selected and shall go through long-term training. In addition, the artistic researchers in China think that there are large differences between grand song of Dong minority and other folk songs no matter on the aspects of singing skills, melody structure or the singing mode and there are relatively more chorus links and there are the euphony types with different parts including high pitch and low pitch. It is the kind of folk heterophony music and possesses relatively high peculiarity and is honored as "living fossil [1]". In 1999, in a program supported by Bai Yansong, he interviewed Mr Yang Zongfu who focuses on the study of Dong minority and displayed the characters and charm of multi-voice and homophony of China which laid good foundation for the development of Chinese music to a large extent.

\section{ClASSIFICATION OF GRAND SONG OF DONG MINORITY}

The grand song of Dong minority mainly refers to the multi-sound song and the grand song of Dong minority can be divided into following several types according to different occasions [2]: 1. vocal grand song. This kind of song mainly manifests the beauty of sound of song and the up and down melody during singing process so as to highlight the melody of songs to the largest extent but has relatively shorter lyrics. The vocal grand song mainly imitates the sound of birds and insects and water flowing beneath a little bridge, etc. The titles are mainly named as birds, beasts and seasons, etc; 2 . soft grand song. It is mainly the incisive expression of the emotions between men and women and its biggest characteristic is relatively more lyrics, shorter prolongation of tune and light and slow melodies; 3 . theory grand song. It is a kind of grand song with no relatively strong fluctuations in the music but the focus more on the lyrics content expression. And its lyrics are mainly sarcasm and praise; 4. narrative grand song. This kind of song is mainly based on story lines to proceed with the dialog among figures which is one of the typical type of "multi-sound talking and singing" and is mainly the kind of pattern of manifestation where one person leads the chorus and other singers sing the fixed accompaniment lyrics at bass. 


\section{ECOLOGICAL ENVIRONMENT FOR GRAND SONG OF DONG MINORITY}

\section{A. Natural Ecological Environment}

The human thoughts trigger thoughts and emotions through outside objects and motivate the musical creativity on that basis. The creation of the grand song of Dong minority is relatively common in Liping, Dong Township and the singer-songwriters are infected by the sound of local insects and hum alone. With the continuous increase of people imitating, it became a kind of fixed melody in local place. For example, the famous Golden Cicada Song was created by the singer by imitating the sound of cicadas and some of the sounds in the nature. This kind of living environment can enable people to generate great emotions and associations on the living environment of themselves and finally transfers the sounds of nature into beautiful singing [3]. Goetschius, the famous foreign musician once said that the ancient and original melodies are reflections of people to the bird tweet and natural awareness of sounds of nature. This guess just proved the formation form of grand song of Dong Minority.

\section{B. Ecological Environment for Folk Culture}

During the development process of grand song of Dong minority, it has its unique development environment and comprehensively manifests the singing in folk culture, language culture and production culture and has major relation with the characters of people, custom and living environment to a large extent. in addition, the grand song of Dong minority is mainly a kind of distinctly important mode for the inheritance of this folk culture. In addition, the grand song of Dong minority was created in Song Dynasty and was the most important form for the affective interaction of people. In the region of Liping in China, on every festival or when receiving guests, there will always be drum-tower grand song show held in a solemn manner to express the sincere welcome. It can be seen that the grand song of Dong Minority is originated from life and it can only manifest the meanings of grand song of Dong minority in the minority folk custom.

\section{PROTECTION AND INHERITANCE OF GRAND SONG OF DONG MINORITY}

\section{A. Enhancing the Publicity of Folk Activities}

During the publicity process of grand song of Dong minority, it requires to adopt some effective measures to enhance the publicity through government which can expand the development space of grand song of Dong minority to a large extent. And on this basis, it also requires to look into the living status of singers and render help to guarantee the singer to the have the ability to organize the teams which can guarantee the continuous development of grand song of Dong minority to a large extent. In addition, it also requires to develop the tourism with the main body of grand song of Dong minority which can not only promote the local economic level but also can promote the popularity of grand song of Dong minority in the society to a large extent and the development of tourism projects will attract the foreign culture which can integrate other culture based on its own culture while expanding the cultural influence of grand song of Dong minority so as to effective integrate various kinds of culture and give play to the creativity of grand song of Dong minority to the maximum extent. This can not only guarantee the effective protection and inheritance of grand song of Dong minority but also plays key role in the inheritance of our national culture. It can also promote the attention on Dong culture of people through the cultural exchange by means of some folk activities.

\section{B. Cultivating Inheritors for the Development of Dong Minority}

During the development process of grand song of Dong minority, it is the most important to look for suitable inheritors which plays key role for the future development of grand song of Dong minority. We can combine the chorus of grand song of Dong minority and the art teams to get together and cultivate the power of youth in the positive activities so as to promote the interests of the youth and enable them to learn the grand song of Dong minority through their apperception and accomplishments [5]. In addition, we shall also cultivate a batch of excellent youth who can go out of home which is mainly because most culture in China has been integrated with business models and urbanization is continuously developing. Therefore, the grand song of Dong minority needs to take in and screen certain things during development process which also requires the youth to correctly select and develop. Therefore, it requires the government and local organizations to conduct effective cultivation to the youth. This can not only guarantee the effective development of grand song of Dong minority but also can promote the professional abilities of grand song of Dong minority to a large extent.

\section{Developing the Exchange Activities in Different Regions}

The region of Dong minority also needs to exchange among different nations with different regions which can not only effectively spreads the grand song of Dong minority but also can promote the emotional exchange and unification of different nations. In addition, there are many people interested in music have deep understanding about the songs of minorities including some of the grand songs of Dong minority. The reason why the spreading grade of grand song of Dong minority is relatively low is that people have less opportunity to contact the music of minorities and they cannot understand the true forms of minority music to a large extent. The acoustics cannot manifest the profound cultural connotations of minority music and cannot truly manifest the true connotations of grand song of Dong minority which requires the effective cultural exchange of different nations for the effective integration of the music of different nations. Only in this way can the youth truly learn the cultural value of different national music so as to establish the sense of pride of themselves. 


\section{Constructing the Protection Mechanism of Inheritors}

During the development and inheritance process of the grand song of Dong minority in Dong minority region of China, the inheritance modes are relatively diversified including inheritance from master to apprentice, family's heritage and community inheritance, etc. Thereinto, the inheritance from master to apprentice mainly conducts oneon-one learning of students of grand song of Dong minority from the masters with participation of activities of grand song of Dong minority during learning process. Generally, the age of masters of grand song of Dong minority is about 65 who mainly teach the songs for the youth and possess relatively high moral integrity and therefore are well respected by local people. The measures for choosing masters when learning the grand song of Dong minority are mainly related to the local culture, etiquette and custom [6]. The family's heritage mainly means that the parents act as the teachers of children for teaching the grand song of Dong minority. There is a widely spread children's song in Dong minority region: whip the wooden horse to get faster, roll over in ash, we like crying when we are little and we shall become good singers when growing up. Thus it can be seen that the parents begin to teach the children to sing when they start talking in Tong minority region of China. However, with the economic development of China, many families rarely teach children to sing which is mainly because many parents go out to work for supporting the family and this indicates that the grand song of Dong minority is gradually lost during the economic development process to a large extent. Because the grandparents become the guardians of kids, it becomes hard to guarantee that the kids have sufficient learning time which also impacts the atmosphere for singing to a large extent. Therefore, the family's heritages are gradually transferring to the mode of community inheritance. The community inheritance is mainly to participate in the activities held in Dong minority regions and learn the grand song of Dong minority in such activities. Under such learning mode, there is no clear learning locations and no fixed learning time and is generally oriented by the youth which is also a kind of effective measure for inheritance to a large extent.

\section{E. Integrating Grand Song of Dong Minority into Tourism Project}

Lunch the folk activities according to the folk customs of Dong minority region and attract the tourists of different nations. For this purpose, we can develop the tourism projects in local places which can not only promote the local economic industry but also can promote the impact of grand song of Dong minority to a large extent and guarantee it to be effectively protected and developed and can also guarantee the effective protection and development. It can also make other nations deeply understand the grand song of Dong minority. On the basis of national economic development, many scenic spots and historical sites have been developed rapidly. Thereinto, some of the folk customs in minorities integrate with the tourism resources during development process which is favorable for the future development of grand song of Dong minority to a large extent. The introduction of grand song of Dong minority in tourism projects can not only bring the refreshing feeling to tourists, but also can expand the impacts of grand song of Dong minority in the society, which is mainly because currently people are living the stressful life and hope to relief the pressure through the environment of folk customs. Thus it can be seen that the integration of the essence and cultural connotations of grand song of Dong minority into the tourism projects can not only satisfy the desire of people on folk customs and can guarantee the effective development of grand song of Dong minority. This is also a kind of protection and inheritance of grand song of Dong minority to a large extent and can manifest the cultural connotations of Dong minority through tourism projects so as to promote the rapid economic development of Dong minority to a large extent. If the economy of Dong minority region can obtain effective development, some of the youth may return home which can effectively promote the local economy to be more prosperous and can guarantee the effective protection and inheritance of grand song of Dong minority to a relatively large extent.

\section{F. Building the Cooperation Platform Between Government and the Public}

During the development process of grand song of Dong minority, the government shall not only grant support on the aspect of behaviors but also shall effectively cooperate with the public so as to build a effective platform between the government and the public and guarantee the good protection and inheritance of grand song of Dong minority. Thereinto, during the cooperation process between the government and the public, it requires a run-in period and requires to utilize the local cultural resources and motivate the autonomy of the public on that basis so as to transfer the cultural resources into the resources favorable for themselves [7]. During the protection and inheritance process of grand song of Dong minority, the support and protection of the government are the most important. This requires the government to adopt effective methods to create favorable opportunities for the grand song of Dong minority to provide the opportunities for the youth going out to inherit the classics, such as construction of official performance sites for the grand song of Dong minority and recording and integration of grand song of Dong minority and keep the original grand song of Dong minority. This not only is of great significance for local people but also has profound meaning for the construction of national social spiritual civilization.

\section{CONCLUSION}

In conclusion, during the development process, the grand song of Dong minority of China shall adopt effective methods to protect and inherit which can not only promote the effective construction of Chinese culture. In addition, the Chinese human ecology shall be effectively protected during development process. As a kind of relatively precious tangible cultural heritage of China, the grand song of Dong minority shall obtain the protection of the whole society and shall be effectively inherited on this basis. Only in this way can Chinese culture be developed in a diversified mode which can not only enrich the cultural life of people but also 
can promote the comprehensive quality of people to a large extent so as to promote the comprehensive development of social culture and lay a good foundation for the development of harmonious society.

\section{REFERENCES}

[1] Zhang Junyu, Wen Ziyi, Shan Wanru, et.al. Path and Thoughts for the Protection and Inheritance of Grand Song of Dong Minority - A Case Study of Tongdao District, Huaihua, Hunan [J]. Shiting, 2018 (1): 190-191 (in Chinese)

[2] Huang Xiangmeng. Several Thoughts on Inheritance and Protection [J] Folk Music, 2017 (5). 21-23 (in Chinese)

[3] Zeng Xiaoqian. Study on the Inheritance Features of Grand Song of Dong Minority [J]. Journal of Kaili University, 2018(1): 45-46 (in Chinese)

[4] Li Xiaorong, Wu Taobo, Dai Rulian, et.al. Study on the Problems existing in Protection of Grand Song of Dong Minority and the Solutions[J]. Journal of Zunyi Normal College, 2017 (6). 24-25 (in Chinese)

[5] Deng Jun. Study on the Inheritance and Protection of Children's Song of Dong Minority- A Case Study of Rongjiang Zaidang Village, Guizhou[J]. Theatrical Family, 2016 (4). 71-71 (in Chinese)

[6] Qin Jiajin. Bring Grand Song of Dong Minority into Large Classes [J]. Comparative Study of Cultural Innovation, 2017 (27).21-22 . (in Chinese)

[7] Wu Bo, Discussion of Development Status of Grand Song of Dong Minority [J]. Chinese Information, 2017 (7): 74-75. (in Chinese). 